\title{
AURC Infinity Observed Normalized by Dose
}

National Cancer Institute

\section{Source}

National Cancer Institute. AURC Infinity Observed Normalized by Dose. NCI Thesaurus.

Code C92355.

The area under the urinary excretion rate curve (AURC) extrapolated to infinity, based on the last observed excretion rate, divided by the dose. 